\title{
HIGHLIGHTS
}

THERAPY

\section{Long-term use of dietary supplements no more effective than placebo in $O A$}

Glucosamine and chondroitin sulphate, dietary supplements popular with individuals who have osteoarthritis (OA), have been shown to be no more efficacious than placebo or the NSAID celecoxib, according to the 2-year follow-up results from the Glucosamine/chondroitin Arthritis Intervention Trial (GAIT) now published in the Annals of the Rheumatic Diseases.

Sawitzke et al. present data from 662 patients with knee OA who were included in the GAIT ancillary structural study. Patients were randomly assigned to receive the dietary supplements administered either singly ( $n=134$ for glucosamine $500 \mathrm{mg}$ three times daily; $n=126$ for chondroitin sulphate $400 \mathrm{mg}$ three times daily) or in combination $(n=129)$, celecoxib $200 \mathrm{mg}$ once daily $(n=142)$ or placebo $(n=131)$ over a period of 24 months in the multicenter, double-blind study. The primary outcome measure was the likelihood of achieving a
$20 \%$ decrease in the Western Ontario and McMaster Universities Arthritis Index (WOMAC) pain score over 2 years, and secondary measures included the level of pain reduction attributable to each agent and the WOMAC function subscore.

Although the scientists observed an improvement in both pain and function, the degree was found to be similar in all treatment groups and no agent was statistically more effective than placebo. Patients receiving celecoxib and glucosamine monotherapy did show the greatest improvement but the results were not considered clinically important.

\section{Shreeya Nanda}

Original article Sawitzke, A. D. et al. Clinical efficacy and safety of glucosamine, chondroitin sulphate, their combination, celecoxib or placebo taken to treat osteoarthritis of the knee: 2-year results from GAIT. Ann. Rheum. Dis. 69, 1459-1464 (2010) 